\title{
Summary and meta-analysis of prospective studies of animal fat intake and breast cancer
}

\author{
Dominik D. Alexander ${ }^{1}$, Libby M. Morimoto ${ }^{2}$, Pamela J. Mink ${ }^{3,4}$ and Kimberly A. Lowe \\ ${ }^{1}$ Exponent Health Sciences Practice, Wood Dale, IL, USA \\ ${ }^{2}$ Exponent Health Sciences Practice, Menlo Park, CA, USA \\ ${ }^{3}$ Exponent Health Sciences Practice, Washington, DC, USA \\ ${ }^{4}$ Rollins School of Public Health, Emory University, Atlanta, GA, USA \\ ${ }^{5}$ Exponent Health Sciences Practice, Bellevue, WA, USA
}

\begin{abstract}
The objective of the present review was to examine the potential association between animal fat intake and breast cancer. We conducted a meta-analysis and review of epidemiological cohort studies, including data reported in the Pooling Project publication of Prospective Studies of Diet and Cancer. Random- and fixed-effects models were utilised to generate summary relative risk estimates (SRRE), and sensitivity and influence analyses were conducted. In the meta-analysis that included data reported in the Pooling Project publication of prospective cohorts $(n 8)$ and subsequent publications of cohort studies (n 3), no significant association was observed comparing the highest category of animal fat intake with the lowest (SRRE 1.03; $95 \%$ CI: 0.76, 1.40). Similarly, no significant association between a $5 \%$ increment of energy from animal fat intake and breast cancer (SRRE 1.02; $95 \%$ CI 0.97, 1.07) was observed in the meta-analysis of these studies. In conclusion, the results of the present quantitative assessment are not supportive of a positive independent association between consumption of animal fat and breast cancer, although findings may be sensitive to the type of dietary instrument used in cohort studies.
\end{abstract}

Animal fat intake: Breast cancer: Meta-analyses

\section{Background}

Early ecological studies, in which strong correlations between high per capita dietary fat consumption and breast cancer incidence and mortality were observed, led to the hypothesis that fat intake was associated with increasing the risk of breast cancer ${ }^{(1-5)}$. Studies using animal models have shown an effect of fat on the development of mammary tumours that is independent of total energy intake. In a meta-analysis of data from 100 animal experiments, dietary fat was found to increase the incidence of mammary tumours in rats and mice ${ }^{(6)}$. Additionally, in vitro experiments have demonstrated that dietary fat acts as a promoter of carcinogenesis ${ }^{(5)}$. Findings from observational studies among human populations, however, have been equivocal, and associations between overall fat consumption or individual types of fat intake and breast cancer risk have been relatively inconsistent ${ }^{(7)}$. Collectively, more positive associations between consumption of fat (total and specific types) and breast cancer have been reported in case-control studies than cohort studies. Findings from case-control assessments, however, may be subject to recall bias and/or selection bias arising when the exposure distribution of the participating cases or controls is not representative of the population of eligible participants from which the study group was sampled. In a 1990 metaanalysis of case-control studies, Howe et al. ${ }^{(8)}$ reported a statistically significant positive association (summary $\mathrm{OR}=1.35$ ) between total fat intake and breast cancer, with the strongest effect observed among postmenopausal women (summary OR $=1.48$ ). In a 1993 meta-analysis of epidemiological studies, Boyd et al. ${ }^{(9)}$ reported a $21 \%$ statistically significant positive association between total dietary fat and breast cancer among case-control studies but no association was found among seven cohort studies for total fat or fat subtypes. In a 10-year update of this analysis, which included data from thirty case-control studies and fourteen cohort studies, summary associations for total fat between case-control studies (summary relative risk estimate $(\mathrm{SRRE})=1 \cdot 14)$ and cohort studies $(\mathrm{SRRE}=1 \cdot 11)$ were more similar, and a significant positive association for

Abbreviations: ER, oestrogen receptor; PR, progesterone receptor; RR, relative risk; SRRE, summary relative risk estimate.

* Corresponding author: Dr Dominik D. Alexander, fax +1 630274 3299, email dalexander@exponent.com 
saturated fat intake was observed among the cohort studies $(\mathrm{SRRE}=1 \cdot 15)^{(10)}$. Despite some positive associations, many publications of large-scale cohort studies have observed weak to modest non-significant associations on both sides of the null value. Smith-Warner et al. (11) evaluated overall dietary fat and specific types of dietary fat in relation to breast cancer based on an analysis of the Pooling Project of Prospective Studies of Diet and Cancer, in which standardised exposure and analytical methodology was implemented and primary data from eight large prospective cohort studies were analysed. The authors observed a weak positive association with substitution of saturated fat for carbohydrate consumption but no evidence of significant positive associations was reported for the highest ( $v$. lowest) intake levels of saturated fat. Moreover, no consistent patterns of positive associations were observed for total fat or any of the other subtypes of fat. In the recent Women's Health Initiative Randomized Controlled Dietary Modification Trial in which the effects of a low-fat diet intervention among postmenopausal aged women were evaluated, a non-significant reduction of breast cancer risk (hazard ratio $=0.91 ; 95 \% \mathrm{CI} 0.83,1.01$ ) among women in the intervention group was observed ${ }^{(12)}$.

Although many studies have evaluated types of dietary fat or fatty acids, such as total fat or saturated and unsaturated fat intake, fewer studies have focused specifically on fat intake from animal sources. The composition of fatty acids differs between animal and vegetable sources, and, thus, may have varying effects on cancer risk, such as breast cancer $^{(13)}$. In the USA, MUFA and SFA are the predominant contributors to animal fat, while PUFA, MUFA and transfatty acids contribute primarily to vegetable fat ${ }^{(13)}$. However, consumption of fish, a source of animal fat, is a primary source of some polyunsaturated fats, such as EPA. Sources of animal fat may include meat, such as from ruminants (cattle or sheep), pork or poultry, or from marine life, eggs or dairy foods. Animal fat from cooking or manufacturing includes tallow, suet, lard and butter ${ }^{(14)}$. In a 1996 publication of the aforementioned Pooling Project, Hunter et al. ${ }^{(15)}$ observed no association comparing the highest with the lowest animal fat intake categories among seven prospective cohort studies. In an update of this analysis (i.e. Smith-Warner et al. ${ }^{(11)}$ ), which included additional cases from four cohorts and data from another cohort, animal fat consumption remained unrelated to breast cancer risk among all women, premenopausal women and postmenopausal women ${ }^{(11)}$. Conversely, in an analysis of the Nurses' Health Study II cohort, animal fat intake largely from red meat and high-fat dairy foods was associated with an increased risk of breast cancer during premenopausal years ${ }^{(13)}$

Recently, some key cohort studies of animal fat intake and breast cancer have been published that have not been examined in previous quantitative assessments ${ }^{(13,16-21)}$. Therefore, to evaluate how the results from these additional cohorts contribute to the current state of the science and to further elucidate the potential relationship between animal fat intake and breast cancer, we conducted a meta-analysis and review of epidemiological cohort studies published to date. The present analysis and review includes an evaluation of the highest compared with the lowest categories of intake, assessments of energy increases from animal fat intake as reported in the epidemiological literature, examinations of potential heterogeneity across studies, and sensitivity and influence analyses.

\section{Materials and methods}

\section{Literature search}

We conducted a MEDLINE literature search using the PubMed interface to identify articles eligible for review. Our search included all articles indexed by PubMed that were published up to 2008. We used unqualified keywords, which are searched as text words in the title, abstract and full journal article. Our search string included terms for breast cancer and dietary fat, including total fat intake, and, specifically, animal fat. The original PubMed search string of 'dietary fat and breast cancer' yielded approximately 1500 articles, for which we applied our inclusion criteria as detailed below. In addition to our PubMed literature search, we examined the bibliographies of review articles and previous quantitative assessments pertaining to fat consumption and breast cancer in an effort to identify all available literature that may not have been identified by our database searches. All data considered for inclusion in our meta-analysis originated from peer-reviewed published articles written in the English language.

\section{Inclusion criteria}

Epidemiological prospective studies that reported results for the association between animal fat intake and female breast cancer were included in the meta-analysis. In addition, we included data from a pooled assessment of prospective cohort studies that reported more data than what was available from individual publications ${ }^{(11)}$. Ecological assessments, correlation studies and other publications of aggregate-level analyses were excluded. Experimental animal studies and mechanistic studies were excluded as well. Case-control studies that evaluated animal fat and breast cancer were obtained; however, our quantitative assessment consisted only of prospective cohort studies because the methodology and reporting of results across case-control studies were heterogeneous. Studies included in the present meta-analysis were required to report data for animal fat as an independent analytical variable, i.e. as a specific nutrient or as a percentage of total energy. Data for analytical variables categorised as total fat, saturated or unsaturated fat were not included because non-animal food sources may have contributed to these exposure variables. Studies were required to report point estimates (i.e. rate ratios) and measures of variability (i.e. $95 \% \mathrm{CI}$ ) for increasing categories of animal fat intake (either in grams, servings or percentage of total energy intake) compared with a lower intake reference category.

\section{Data extraction}

We extracted qualitative information and quantitative data from each study that met the criteria for inclusion. Specifically, we abstracted information pertaining to the 
study population, geographic location of the study, methods of dietary exposure ascertainment, definitions of animal fat, analytical comparison (i.e. the exposure contrast), years and duration of follow-up, number of exposed cases, rate ratios and $95 \%$ CI for each incremental category of intake, factors that were adjusted or controlled for in the analysis, and the likelihood of study population overlap across multiple analyses of the same cohort. Two reviewers (D. D. A. and L. M. M.) extracted individual study information independently as part of our quality-control process. As mentioned previously, we abstracted data for a variable categorised and analysed as animal fat or fat from animal or meat sources, and we did not extract data for variables categorised as total fat, saturated fat or unsaturated fat. This was done to ensure homogeneity within the primary exposure variable.

A thorough review of each article was conducted to identify cohorts that may have been analysed in multiple publications. If results for the same cohort were reported in multiple publications, we based our inclusion of data on (a) the size of the study population, (b) the duration of follow-up with an emphasis on the most recent publication with the longest follow-up, (c) classification and analytical categorisation of animal fat intake, and (d) level of control for potential confounding factors. We used pooled data from a 2001 publication by Smith-Warner et al. ${ }^{(11)}$ in one of our high $v$. low intake meta-analysis models. Their publication was an analysis of the Pooling Project of Prospective Studies of Diet and Cancer, which is an international consortium of cohort studies with the goal of analysing diet and cancer associations using standardised criteria across studies (http://www.hsph.harvard.edu/poolingproject/about. html). Studies that had accrued at least 200 cases of breast cancer, administered a comprehensive FFQ at baseline, and used a validated dietary assessment instrument (or one that was closely related) were included in the Pooling Project analysis. Specifically, Smith-Warner et al. ${ }^{(11)}$ analysed primary data from eight individual study cohorts from North America and Western Europe, contributing 7379 cases of incident breast cancer. Their analyses were adjusted for numerous factors, including hormonal, lifestyle and anthropometric variables. Although eight study cohorts were included in the pooled analysis ${ }^{(11)}$, not all of the cohorts were represented by individual publications reporting results specifically for animal fat and breast cancer. For example, the Pooling Project analysis included data from the Canadian National Breast Screening Study (Howe et al. ${ }^{(22)}$ ); however, data specifically for animal fat was not reported in an independent publication representing this cohort. Thus, in our first meta-analysis model, to avoid study population overlap, we used the pooled estimates from Smith-Warner et al. (11) while excluding data from articles consisting of analyses of individual study cohorts that were evaluated in the Pooling Project. Specifically, we meta-analysed data from the Pooling Project ${ }^{(11)}$, the Japan Collaborative Cohort Study ${ }^{(17)}$, the Hormones and Diet in the Etiology of Breast Cancer Study ${ }^{(18)}$ and the Nurses' Health Study II ${ }^{(13)}$. In our second meta-analysis model, we removed data from the Pooling Project analysis and included relevant data from the articles (where applicable) of study populations that were analysed by Smith-Warner et al. ${ }^{(11)}$. Individual publications representing the Netherlands Cohort Study ${ }^{(23)}$, the Adventist Health Study ${ }^{(24)}$ and the New York State cohort $^{(25)}$ reported data for animal fat and breast cancer that had been included in the Pooling Project.

Several publications of analyses of the Nurses' Health Study cohorts were identified and reviewed but not all were included in the meta-analysis models because of likely population overlap and/or evaluations of dietary characteristics during unique time periods (i.e. pre-school, adolescence $)^{(16,20,21,26)}$. In a 2003 publication of the Nurses' Health Study II, animal fat intake was assessed among premenopausal women (10\% of women became postmenopausal during follow-up) aged 26-46 years who were followed between 1991 and 1999(13), and data from this study were included in our meta-analysis model with the Pooling Project data. In a recent publication of an analysis of the National Institutes of Health-AARP Diet and Health Study, Thiébaut et al. ${ }^{(19)}$ reported results data 'in-text' for a doubling of percentage energy from animal fat; however, not enough data were provided to be included in the present meta-analysis. The characteristics of studies evaluated in the present assessment are reported in Table 1.

\section{Statistical analysis}

Meta-analyses comparing the highest intake category with the lowest (or referent) intake category were conducted. Heterogeneity by menopausal status was assessed in our sensitivity analyses. In addition, we reviewed the patterns of associations across studies for increments of $5 \%$ of energy from animal fat if these data were reported in the individual studies. Data for this analytical metric were reported in Smith-Warner et al. ${ }^{(11)}$ for eight individual cohorts. We used the method proposed by Greenland \& Longnecker $^{(27)}$ to estimate the relative risk (RR) of a $5 \%$ increment of animal fat intake in one study ${ }^{(17)}$ that reported categories of percentage energy from animal intake.

Random-effects models were used to calculate SRRE, $95 \% \mathrm{CI}$ and corresponding $P$ values for heterogeneity. These results were compared with results calculated from fixed-effects models. The estimates of the individual studies were weighted based on the inverse of the variance, which is related to the sizes of the study populations. In our 'one study removed' sensitivity analyses, the relative influence of each study on the model-specific SRRE was examined by generating an SRRE based on all studies in a particular model, followed by the removal of one study at a time in order to compare the overall SRRE with SRRE from models that had one study removed. The presence of publication bias was assessed visually by examining a funnel plot measuring the standard error as a function of effect size, as well as performing Begg \& Mazumdar's rank correlation test and Egger's regression method ${ }^{(28)}$. These tests for publication bias, however, are not particularly powerful in an assessment of relatively few studies such as the present analysis. All statistical analyses were performed using STATA (version 10.0; College Station, TX, USA), Comprehensive Meta-Analysis (version 2.2.046; http://www. meta-analysis.com/; Biostat, Inc., Englewood NJ, USA) and Episheet spreadsheets for the analysis of epidemiological data (http://members.aol.com/krothman/episheet.xls). 


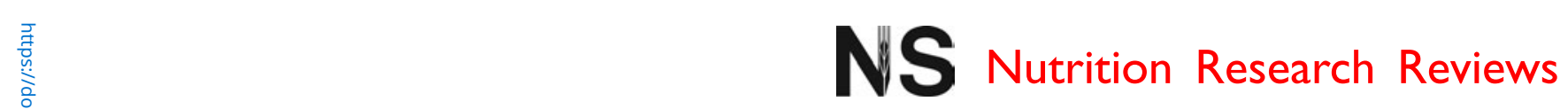

Table 1. Summary of cohort studies of animal fat and female breast cancer

\begin{tabular}{lc} 
Author and year & Study cohort \\
\hline Pooled analysis of prospective cohort studies \\
Smith-Warner & Pooling Project of \\
et al. $(2001)^{(11)}$ & Prospective \\
(update of & Studies of Diet \\
Hunter et al. & and Cancer \\
$\left.1996^{(15)}\right)$ &
\end{tabular}

Relative

\begin{tabular}{|c|c|}
\hline & Total breast \\
\hline
\end{tabular}

Statistical adjustment

\section{$1976-97$}

7329

Quartiles of intake

Pooled

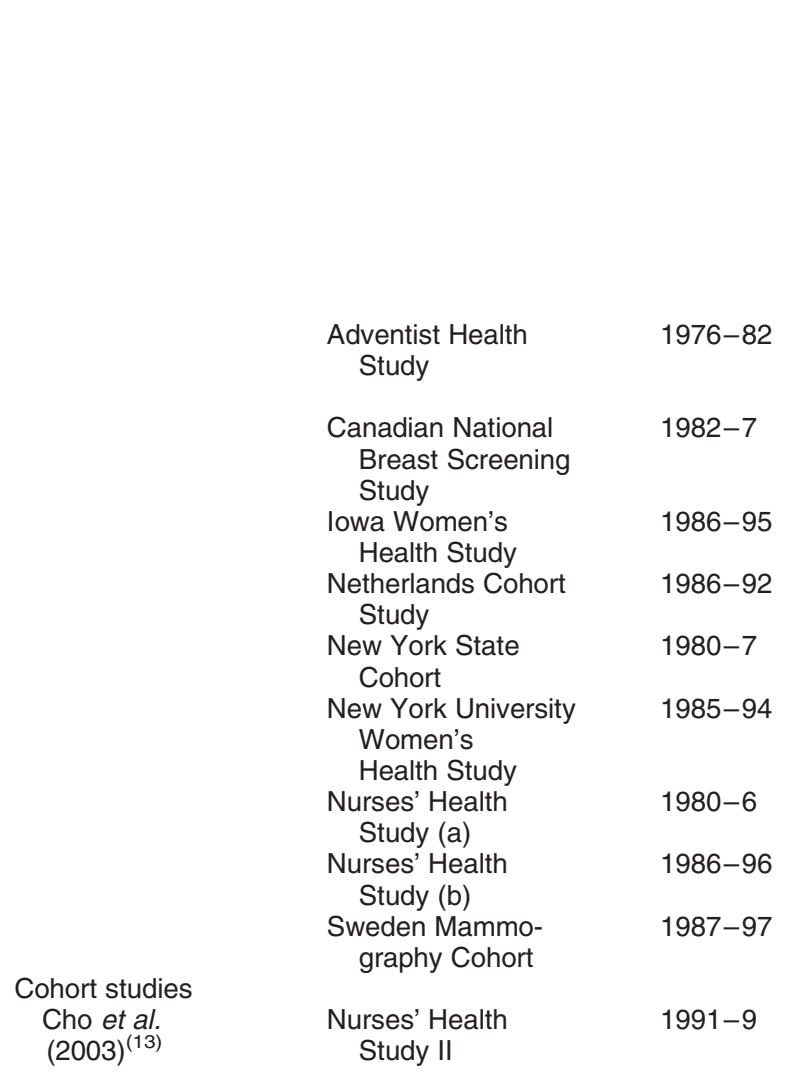

\begin{tabular}{|c|c|}
\hline 2 v. 1 (all women) & $0.98(0.88,1.10)$ \\
\hline $3 v .1$ & $0.93(0.82,1.04)$ \\
\hline 4 v. 1 & $0.96(0.84,1.09)$ \\
\hline $\begin{array}{l}5 \% \text { of energy } \\
\text { increase from } \\
\text { animal fat }\end{array}$ & Pooled \\
\hline All women & $1.01(0.96,1.06)$ \\
\hline Premenopausal & $1.01(0.91,1.12)$ \\
\hline Postmenopausal & $0.99(0.95,1.03)$ \\
\hline $\begin{array}{l}5 \% \text { of energy } \\
\text { increase from } \\
\text { animal fat }\end{array}$ & $1.10(0.84,1.45)$ \\
\hline $\begin{array}{l}5 \% \text { energy increase } \\
\text { from animal fat }\end{array}$ & $1.10(0.97,1.25)$ \\
\hline $\begin{array}{l}5 \% \text { energy increase } \\
\text { from animal fat }\end{array}$ & $1 \cdot 10(1.02,1 \cdot 18)$ \\
\hline $\begin{array}{l}5 \% \text { energy increase } \\
\text { from animal fat }\end{array}$ & $1.02(0.93,1.11)$ \\
\hline $\begin{array}{l}5 \% \text { energy increase } \\
\text { from animal fat }\end{array}$ & $0.88(0.77,1.00)$ \\
\hline $\begin{array}{l}5 \% \text { energy increase } \\
\text { from animal fat }\end{array}$ & $1.01(0.91,1.12)$ \\
\hline $\begin{array}{l}5 \% \text { energy increase } \\
\text { from animal fat }\end{array}$ & $0.99(0.94,1.04)$ \\
\hline $\begin{array}{l}5 \% \text { energy increase } \\
\text { from animal fat }\end{array}$ & $0.96(0.90,1.02)$ \\
\hline $\begin{array}{l}5 \% \text { energy increase } \\
\text { from animal fat }\end{array}$ & $\begin{array}{l}\text { Data not } \\
\quad \text { available }\end{array}$ \\
\hline Premenopausal & \\
\hline
\end{tabular}

23 v. $12 \%$ median intake of energy
$1.33(1.02,1.73)$
Percentage energy from protein, percentage energy from alcohol, age at menarche, parity, age at birth of first child, menopausa status at diagnosis (all women), postmenopausal hormone use, oral contraceptive use, history of benign breast disease, family history of breast cancer, smoking status, education, BMI, BMI-menopausal status interaction term (all women), height, fibre intake, vegetable fat
Age, calendar year of interview, smoking, height, parity, age at first birth, BMI, age at menarche, family history of breast cancer, history of benign breast disease, oral contraceptive use, menopausal status, alcohol intake, energy intake, vegetable fat 


\begin{tabular}{|c|c|c|}
\hline $\begin{array}{l}\text { Frazier et al. } \\
(2004)^{(21)}\end{array}$ & $\begin{array}{l}\text { Nurses' Health } \\
\text { Study II }\end{array}$ & $1989-98$ \\
\hline $\begin{array}{l}\text { Graham et al. } \\
(1992)^{(25)}\end{array}$ & New York & $1980-7$ \\
\hline $\begin{array}{l}\text { Holmes et al. } \\
(1999)^{(26)}\end{array}$ & $\begin{array}{l}\text { Nurses' Health } \\
\text { Study }\end{array}$ & $1980-94$ \\
\hline $\begin{array}{l}\text { Kim et al. } \\
(2006)^{(16)}\end{array}$ & $\begin{array}{l}\text { Nurses' Health } \\
\text { Study }\end{array}$ & $1980-2000$ \\
\hline $\begin{array}{l}\text { Michels et al. } \\
(2006)^{(20)} \dagger\end{array}$ & $\begin{array}{l}\text { Nurses' Health } \\
\text { Study I and II }\end{array}$ & $\begin{array}{c}1976 \text { (NHS I), } \\
1989 \text { (NHSII) } \\
-1993\end{array}$ \\
\hline $\begin{array}{l}\text { Mills et al. } \\
(1989)^{(24)}\end{array}$ & $\begin{array}{l}\text { Seventh-Day } \\
\text { Adventist Cohort }\end{array}$ & $1976-82$ \\
\hline $\begin{array}{l}\text { Sieri et al. } \\
(2002)^{(18)} \dagger\end{array}$ & $\begin{array}{l}\text { Hormones and Diet } \\
\text { in the Etiology of } \\
\text { Breast Cancer } \\
\text { Study }\end{array}$ & $\begin{array}{c}1987-92 \\
\quad \text { (cases } \\
\text { identified } \\
\text { before } \\
1995 \\
\text { average } \\
5.5 \text { years } \\
\text { follow-up) }\end{array}$ \\
\hline
\end{tabular}

Adolescent diet

359

2956

All women

Premenopausal

Postmenopausal

3537

Postmenopausal

$5 \%$ energy increase from animal fat

Preschool diet

Mean intake in $19.3 \mathrm{~g} / \mathrm{d}$

4th quartile of

percentage of

energy from

animal fat

v. 1st quartile

Postmenopausal

follow-up) quintiles: $62.3 \mathrm{v}$

Age, time period, total energy intake, height, parity and age at first birth, BMI at age

18 years, age at menarche, family history of breast cancer, history of benign breast disease, menopausal status, alcohol

intake, contraceptive use, weight gain since age 18 years

$1 \cdot 12(0 \cdot 78,1 \cdot 61)$

Age, education

$1 \cdot 12(0.78,1.61)$

Age, energy, energy-adjusted vitamin A intake, alcohol intake, time period, height, parity, age at first birth, weight change since age 18 years, BMI at age 18 years, age at menopause, menopausal status, use of HRT, family history, benign breast disease, age at menarche

$1.98(0.96,1.01)$

$0.98(0.94,1.02)$

Age, energy, alcohol intake, time period, height, parity, age at first birth, weight change since age 18 years, $\mathrm{BMI}$ at age 18 years, age at menopause, use of HRT, family history of breast cancer, benign breast disease, age at menarche, vegetable fat

$0.97(0.94,1.00)$

$1.05(0.75,1.48)$

$1 \cdot 21(0 \cdot 81,1 \cdot 81)$

Age, age at menarche, parity, age at first birth, family history, adult BMI, total energy intake

Age at entry, age at first live birth, age at menarche, menopausal status, history of benign breast disease, maternal history of breast cancer, educational attainment, BMl

Matched: age, daylight-saving period at recruitment, recruitment centre, recruitment date. Adjusted: parity, place of birth, level of education, total fat

$$
\begin{aligned}
& 36.3-75.9 \\
& \text { v. }<27.6 \mathrm{~g} / \mathrm{d}
\end{aligned}
$$

Non-alcohol energy 


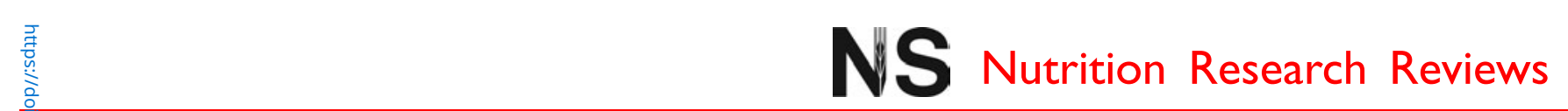

Table 1. Continued

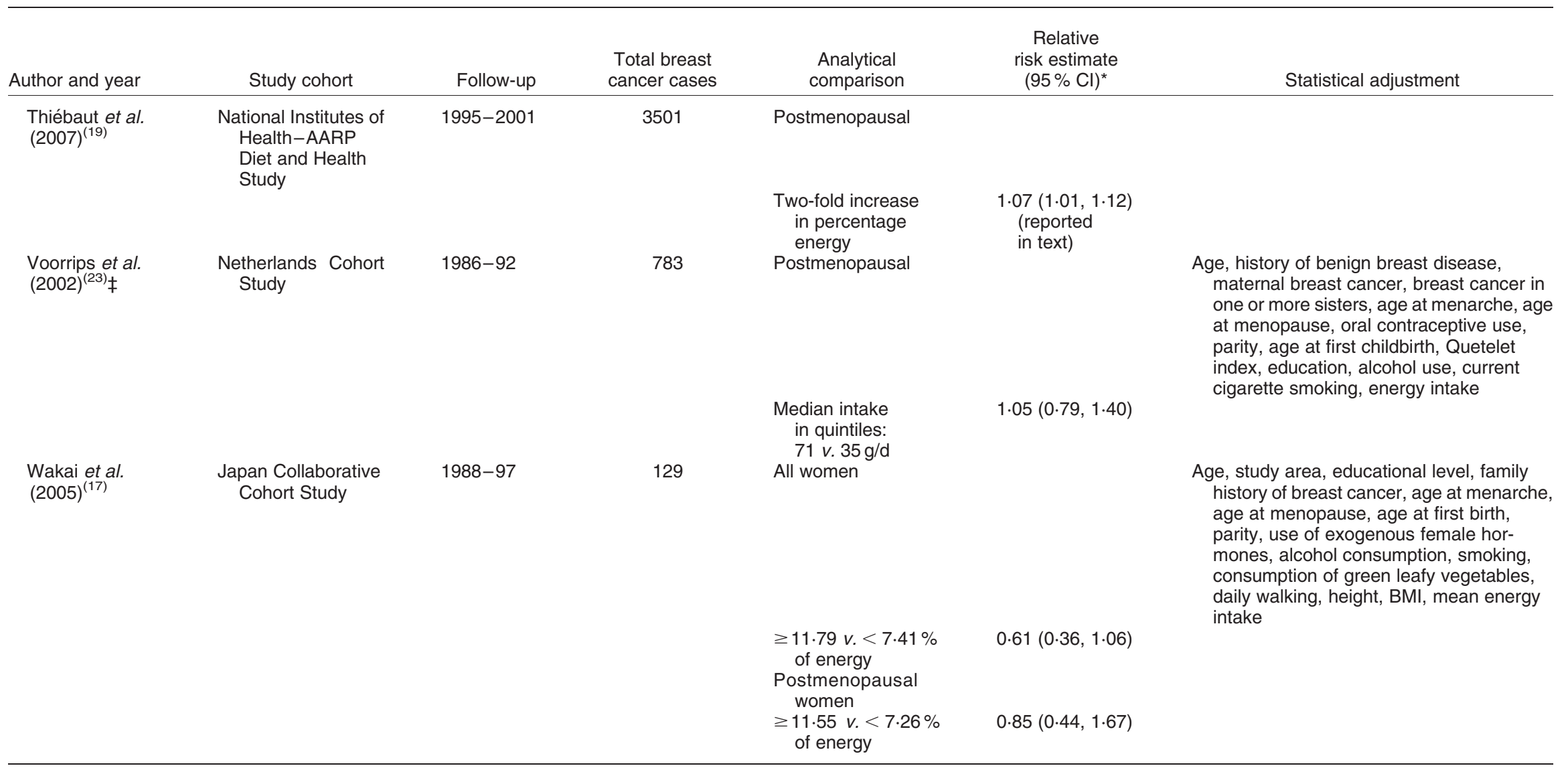

HRT, hormone replacement therapy.

* Highest $v$. lowest intake comparison unless otherwise noted.

† Nested case-control study.

$\ddagger$ Case-cohort study. 

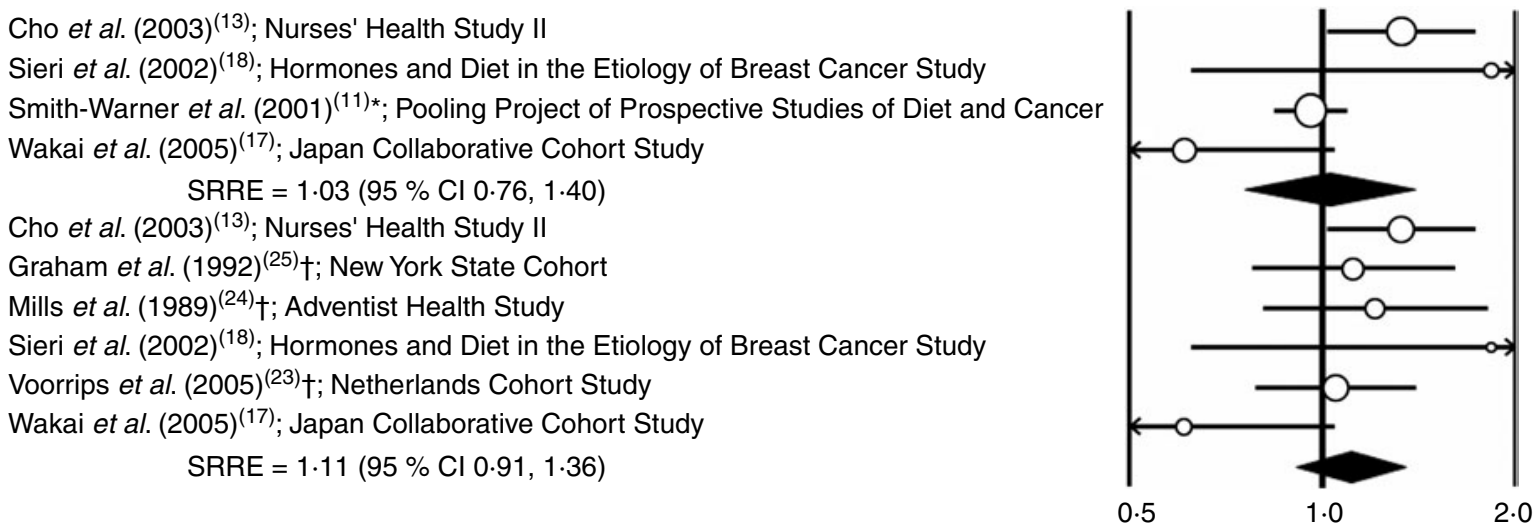

Fig. 1. Meta-analysis of cohort studies of animal fat intake and breast cancer. * Pooled analysis of eight prospective cohort studies: Adventist Health Study, Canadian National Breast Screening Study, lowa Women's Health Study, Netherlands Cohort Study, New York State Cohort, New York University Women's Health Study, Nurses' Health Study (1980-6), Nurses' Health Study (1986-96) and Sweden Mammography Cohort. † Study population overlap with Smith-Warner et al. ${ }^{(11)}$. RR, relative risk; SRRE, summary relative risk estimate.

The utilisation of independent analytical programs allowed for the validation of calculations.

\section{Results}

In the meta-analysis that included data from the Pooling Project of Prospective Cohorts $(n 8)$ and data from subsequent publications of cohort studies ( $n 3$ ), no significant association was observed comparing the highest category of animal fat intake with the lowest (SRRE 1.03; $95 \%$ CI $0.76,1.40$ ), based on analysis using a random-effects model (Fig. 1). The random-effects model gave only $41 \%$ of relative weight to the large pooled analysis by Smith-Warner et al. ${ }^{(11)}$. In contrast, this study was more appropriately given $76 \%$ of the relative weight in the fixed-effects model, resulting in an SRRE of 1.01 (95\% CI 0.90, 1.13). However, significant heterogeneity was observed across the estimates in this model $(P$ value for heterogeneity $=0.026)$. In our 'one study removed' sensitivity analyses, variability was reduced modestly by the exclusion of Cho et al. ${ }^{(13)}$ ( $P$ value for heterogeneity after exclusion $=0 \cdot 132$ ).

After removal of the Pooling Project data, six individual publications ${ }^{(13,17,18,23-25)}$ reported data specifically for incremental categories of animal fat intake and breast cancer (Fig. 1). Publications for cohorts included in the Pooling Project (i.e. Canadian National Breast Screening Study, the Iowa Women's Health Study, the Sweden Mammography Cohort, New York University Women's Health Study and the Nurses' Health Study I) did not report data that could be included in the second metaanalysis shown in Fig. 1. Compared with the model that included Smith-Warner et al. ${ }^{(11)}$ the summary association for the model of six individual publications was slightly stronger in magnitude in the positive direction but was not statistically significant (random-effects SRRE $1.11 ; 95 \%$ CI $0.91,1.36)$. The SRRE for the fixed-effects model was similar $(1.13 ; 95 \%$ CI $0.93,1.32 ; P$ value for heterogeneity $=0 \cdot 178)$. The study by Cho et al. ${ }^{(13)}$ contributed the greatest amount of relative weight (i.e. $26 \%$ ), and removal of this study from the model resulted in a random-effects SRRE of $1.04(95 \%$ CI $0.83,1.31)$ with slightly less variability $(P$ value for heterogeneity $=0.237$ ).

Results for a $5 \%$ of energy (from animal fat intake) increment across cohorts are summarised in Fig. 2. SmithWarner et al. ${ }^{(11)}$ reported data for eight cohorts using standardised exposure and analytical procedures, and, overall, virtually no association with breast cancer was reported among all women or pre- or postmenopausal women (Fig. 2). In regards to statistical significance, a $10 \%$ statistically significant increased risk of breast cancer was found among women in the Iowa Women's Health Study and a marginally significant $12 \%$ reduced risk was observed among women in the New York State cohort with a $5 \%$ increase in total energy from animal fat. Cho et al. ${ }^{(13)}$ reported a statistically significant $12 \%$ increased risk of breast cancer among premenopausal women in the Nurses' Health Study II cohort. Non-significant inverse associations among all women and postmenopausal women were reported by Wakai et al. ${ }^{(17)}$ in an analysis of the Japan Collaborative Cohort Study (Fig. 2). Using the individual study data as reported in Smith-Warner et al. ${ }^{(11)}$ for a $5 \%$ increment of energy from animal fat, we created a meta-analysis model incorporating additional data. We replaced the Nurses' Health Study data from Smith-Warner et al. ${ }^{(11)}$ with data from Kim et al. ${ }^{(16)}$. Thus, when data from Kim et al. ${ }^{(16)}$, Cho et al. ${ }^{(13)}$ and Wakai et al. ${ }^{(17)}$ were combined with the remaining data reported in Smith-Warner et al. ${ }^{(11)}$, the summary association for a $5 \%$ increment of animal fat intake was 1.02 (95\% CI 0.97, 1.07) (results not shown).

In analyses examining the relationship of animal fat and breast cancer by menopausal status, the SRRE for the highest compared with the lowest intake categories of animal fat was 1.10 (95\% CI 0.91, 1.32) (results not shown) based on data from five cohorts consisting solely ${ }^{(18,23,25)}$ or primarily ${ }^{(17,24)}$ of postmenopausal women. No association 


\begin{tabular}{|c|c|c|c|}
\hline udy cohort & $\begin{array}{l}\text { Rate } \\
\text { ratio }\end{array}$ & $\begin{array}{l}\text { Lower } \\
\text { limit }\end{array}$ & $\begin{array}{c}\text { Upper } \\
\text { limit }\end{array}$ \\
\hline Adventist Health Study & $1 \cdot 10$ & 0.84 & 1.45 \\
\hline Canadian National Breast Screening Study & $1 \cdot 10$ & 0.97 & $1 \cdot 25$ \\
\hline lowa Women's Health Study & $1 \cdot 10$ & 1.02 & $1 \cdot 18$ \\
\hline Nurses' Health Study (1980-6) & 0.99 & 0.94 & 1.04 \\
\hline Nurses' Health Study (1986-96) & 0.96 & 0.90 & 1.02 \\
\hline Netherlands Cohort Study & 1.02 & 0.93 & $1 \cdot 11$ \\
\hline New York State Cohort & 0.88 & 0.77 & 1.00 \\
\hline ew York University Women's Health Study & 1.01 & 0.91 & $1 \cdot 12$ \\
\hline Pooled* & 1.01 & 0.96 & 1.06 \\
\hline Pooled: postmenopausal ${ }^{\star}$ & 0.99 & 0.95 & 1.03 \\
\hline Pooled: premenopausal* & 1.01 & 0.91 & $1 \cdot 12$ \\
\hline Cho et al. $2003^{(13)} \mathrm{NHS}$ II: premenopausal & $1 \cdot 12$ & 1.03 & $1 \cdot 22$ \\
\hline Kim et al. $2006^{(16)} \dagger \mathrm{NHS}$ I: postmenopausal & 0.97 & 0.94 & 1.00 \\
\hline Wakai et al. $2005^{(17)} \mathrm{JACC}$ & 0.96 & 0.92 & 1.01 \\
\hline Wakai et al. $2005^{(13)} \mathrm{JACC}$ : postmenopausal & 0.99 & 0.94 & 1.05 \\
\hline
\end{tabular}

Fig. 2. Summary of associations for each $5 \%$ increase in energy from animal fat and breast cancer (findings from prospective studies). ${ }^{*}$ As reported by Smith-Warner et al. ${ }^{(11)}$. † The study population was followed for 20 years (1980-2000); population overlap with Smith-Warner et al. ${ }^{(11)}$. RR, relative risk; NHS, Nurses' Health Study; JACC, Japan Collaborative Cohort Study.

for a $5 \%$ increase in energy from animal fat, however, was observed among postmenopausal women in the pooled analysis of eight prospective cohort studies (pooled RR $0.99 ; 95 \%$ CI $0.95,1.03)^{(11)}$. In a recent publication ${ }^{(16)}$ of the Nurses' Health Study cohort (some population overlap with Smith-Warner et al. ${ }^{(11)}$ ), which included 20 years of follow-up, a marginally significant inverse association was reported for an increment of $5 \%$ energy from animal fat (RR 0.97; $95 \%$ CI 0.94, 1.00). Cho et al. ${ }^{(13)}$, in an analysis of the Nurses' Health Study II cohort, ascertained animal fat intake among premenopausal women aged $26-46$ years at baseline. The authors observed marginal and statistically significant positive associations ranging between 1.28 and 1.54 for all quintiles of animal fat intake ( $P$ value for trend $=0.002$ ). Approximately $10 \%$ of these women became postmenopausal during follow-up, and the RR restricted to women who remained premenopausal throughout follow-up was 1.24 (95\% CI 0.94, 1.64). In contrast, no association (RR 1.01; $95 \%$ CI 0.91, 1.12) was reported among premenopausal women in the Pooling Project analysis by Smith-Warner et al. ${ }^{(11)}$.

There was no indication of publication bias in our funnel plot or statistical assessments.

\section{Discussion}

The potential relationship between dietary fat and risk of breast cancer is one of scientific debate ${ }^{(7)}$. In particular, there is considerable scientific interest in determining whether specific types of fat increase or decrease the risk of breast cancer. Thus, we focused this assessment on animal fat as a specific nutrient and/or as a percentage of energy based on consumption of fat from animal sources.

The largest publication to date that assessed the relationship between animal fat intake and breast cancer using primary source data was the 2001 publication of the Pooling Project analysis by Smith-Warner et al. ${ }^{(11)}$ for which over 7000 incident cases of invasive breast cancer among women from eight prospective cohorts were analysed. No increase in breast cancer was observed after adjustment for numerous demographic, anthropometric, lifestyle and dietary factors, including mutual adjustment for vegetable fat intake. In the present assessment, data from three additional cohort studies were combined with the data from Smith-Warner et al. ${ }^{(11)}$. Cho et al. ${ }^{(13)}$ analysed premenopausal women from the Nurses' Health Study II cohort, Sieri et al. ${ }^{(18)}$ analysed a cohort of postmenopausal women from Northern Italy, and Wakai et al. ${ }^{(17)}$ was a multicentre cohort conducted in Japan. Combining high v. low intake data from these studies with the data from SmithWarner et al. ${ }^{(11)}$ resulted in a non-significant summary association close to the null value. Furthermore, no statistically significant positive association was observed for an increment of $5 \%$ energy from animal fat intake and breast cancer. However, in a recently published analysis of fat consumption and breast cancer among 188736 postmenopausal women in the National Institutes of Health-AARP Diet and Health Study, Thiébaut et al. ${ }^{(19)}$ reported (in text only) a hazard ratio of 1.07 (95\% CI $1.01,1.12)$ for a twofold increase in percentage energy from animal fat. Because results data were not reported in quantile format or as a $5 \%$ increase in energy as with many of the other studies, and because data were not presented in a format that allowed for standardisation, we could not meta-analyse their data.

Findings from case-control studies that evaluated intake of animal fat and breast cancer have been relatively inconsistent; while increased risks have been reported in some case-control investigations ${ }^{(29-33)}$, most associations have not been statistically significant and some studies have observed inverse associations ${ }^{(34-36)}$. Moreover, consistently 
valid findings may be difficult to achieve from case-control studies of dietary factors because of the potential for systematic error (i.e. recall bias and/or selection bias) ${ }^{(37)}$. In addition, the analytical classification of animal fat consumption varied substantially across case-control studies (for example, foods high in animal fat, lard, visible fat on meat), as did the type of exposure contrast (for example, servings per week, g per $\mathrm{d}$, little $v$. never) and level of adjustment for potential confounding factors. Thus, the present assessment was based on data from prospective studies.

Diet early in life may contribute to subsequent risk of cancer, especially for cancers associated with adolescent development and/or reproductive cancers, such as breast cancer. Although limited to few studies, the currently available epidemiological evidence has not provided support for an association between the consumption of animal fat early in life and risk of breast cancer. Michels et al. ${ }^{(20)}$ evaluated the potential effects of preschool diet on subsequent breast cancer risk later in life in a case-control study nested within the Nurses' Health Study I and II cohorts. The mothers of participants in the Nurses' Health Study cohorts were asked about their daughters' perinatal and early life events, including information on foods consumed by the daughter during preschool years using a thirty-food item questionnaire. No significant elevation of breast cancer risk later in life was observed based on the highest preschool intake of animal fat compared with the lowest (RR 1.05; $95 \%$ CI 0.75, 1.48). Recall bias was an inherent limitation, as exposure occurred during 1924 to 1970. In an analysis of diet slightly later in life (i.e. adolescence), a life period that may be affected by micronutrient intake during adolescent growth, participants of the Nurses' Health Study II cohort were asked to complete a questionnaire regarding diet during high school $^{(21)}$. A non-significant positive association was reported for animal fat intake (RR $1 \cdot 12$; $95 \%$ CI 0.78 , 1.61) while non-significant inverse associations were observed for total, vegetable, saturated, monounsaturated and polyunsaturated fat.

Kim et al. ${ }^{(16)}$, in their publication of a 20-year follow-up of women in the Nurses' Health Study, analysed the potential relationship between animal fat intake and risk of breast cancer by oestrogen receptor (ER) and progesterone receptor (PR) status. Non-significant inverse associations between animal fat and postmenopausal breast cancer ranging between 0.96 and 0.99 were reported for the combinations of receptor status (i.e. $\mathrm{ER}^{+} / \mathrm{PR}^{+}, \mathrm{ER}^{+} / \mathrm{PR}^{-}$, $\left.\mathrm{ER}^{-} / \mathrm{PR}^{+}, \mathrm{ER}^{-} / \mathrm{PR}^{-}\right)$. Cho et al. ${ }^{(13)}$ analysed a subset of premenopausal women in the Nurses' Health Study II cohort and the authors reported statistically significant positive associations for a $5 \%$ increase in energy from animal fat ranging between 1.17 and 1.20 for $\mathrm{PR}^{+}, \mathrm{ER}^{+}$and $\mathrm{ER}^{+} / \mathrm{PR}^{+}$breast cancer. However, non-significant associations ranging between 1.04 and 1.11 were reported for $\mathrm{PR}^{-}, \mathrm{ER}^{-}$and $\mathrm{ER}^{-} / \mathrm{PR}^{-}$breast cancer. In their other evaluations of effect modification, significant interactions were observed for $\geq 4$ years of oral contraceptive use and age of 25 years or greater at first birth.

Some important biological, methodological and analytical factors should be considered when interpreting results from the present assessment. The hypothesised mechanism(s) involved between dietary fat and breast cancer remain obscure. In fact, no compelling mechanism relating animal fat to increasing the risk of breast cancer has emerged from the scientific literature ${ }^{(13)}$. Further, the ratio of fatty acid composition varies by source of food, and different international cultures can have distinct patterns of diet that may modify the sources and nature of fat in the diet. In addition, fats from animal sources may have a heterogeneous array of unsaturated lipids, and fats from non-animal sources may contain a variety of unsaturated as well as saturated lipids. Thus, there is overlap in terms of the fractions and types of fatty acids between classification sources. Indeed, there is a common misperception that the majority of fatty acids in beef are saturated; however, approximately half of the fatty acids are monounsaturated, the same type found in olive oil ${ }^{(38)}$. Even though there is overlap between sources of fat, large-scale investigations of specific fatty acids have not produced consistent evidence of positive associations for breast cancer, and analyses that isolated fat from animal sources and fat from vegetable sources have been virtually the same ${ }^{(11)}$.

As mentioned in the section on inclusion criteria, the focus of the present assessment was to evaluate the epidemiological studies of animal fat (i.e. an epidemiological variable reflecting fat intake from animal sources) and risk of female breast cancer. Although we made an effort to ensure homogeneity within the exposure variable, that is, isolating the effects of animal fat as a specific dietary fat constituent, there may be some within-category variability as well by specific sources of animal fat. For example, $n-3$ fatty acids, such as EPA and DHA, are abundant in fish fat, and have been shown to inhibit the proliferation of breast cancer cell lines in vitro and to suppress the progression of the tumours in animal experiments by inhibiting eicosanoid biosynthesis from arachidonic acid or by activating PPAR- $\gamma^{(17,39)}$. The study by Wakai et al. ${ }^{(17)}$ was conducted on a population that consumed a high amount of fish; removal of data from this study in the meta-analysis resulted in an SRRE of 1.15 but this finding was not statistically significant (95\% CI 0.85, 1.55). Experimental and in vitro studies have shown that conjugated linoleic acid (CLA), which is a naturally occurring trans-fat commonly found in ruminant animal sources such as beef and lamb or in dairy products, may protect against mammary carcinogenesis ${ }^{(40)}$. However, epidemiological studies of CLA and breast cancer have been mixed ${ }^{(41)}$.

Meta-analyses, distinct from pooled analyses (i.e. individual-level or raw data are analysed) are limited to analyses of results data as reported within and across publications. It is likely that many studies that evaluated fat intake and breast cancer did not examine fat solely from animal sources; thus, differential reporting of dietary factors may influence summary associations across the literature. In addition, the ability to comprehensively analyse hypotheses that have emerged relatively recently, such as the effect of diet during early periods in life or the potential association between dietary fat and breast cancer subgroups (for example, menopausal status, ER status), is incomplete, and additional studies are necessary to fully examine these hypotheses. 
The present quantitative assessment was restricted to data from prospective studies. Compared with case-control studies, cohort studies may theoretically eliminate differential recall of prior dietary history between cases and controls. That is, exposure (i.e. consumption of animal fat and other factors) is ascertained before disease occurrence. Cohort studies, as with case-control studies, rely upon self-reported techniques for measuring dietary intake, and the potential for measurement error cannot be eliminated. The most common dietary assessment method in cohort studies of diet and cancer is the FFQ, which is the method utilised to ascertain animal fat intake in all of the studies included herein. Although FFQ may not be overly accurate in estimating the actual amount of intake, this instrument is considered to be accurate in ranking individuals by their usual frequency of consumption and determining patterns of intake among populations ${ }^{(42)}$. However, Bingham et al. ${ }^{(43)}$ showed that analysing dietary fat data ascertained from a $7 \mathrm{~d}$ food diary resulted in considerably stronger positive associations for breast cancer compared with data derived from FFQ. In a subsequent, and larger, comparison of dietary ascertainment methodologies, a similar finding was observed; associations for dietary fat and breast cancer were stronger when data from food diaries were analysed compared with $\mathrm{FFQ}^{(44)}$. In a recent methodological article, it was suggested that the performance of the dietary instrument (for example, FFQ $v$. food diary) used to investigate fat intake and breast cancer may have an impact on the interpretation of this potential relationship if measurement errors are prevalent ${ }^{(45)}$. The authors concluded that there may be a modest, but real, association between fat intake and breast cancer, but this relationship is not yet conclusive. This suggestion, however, was not specific to 'animal fat' as a dietary variable.

In summary, the collection of available epidemiological evidence from prospective studies does not support an independent association between animal fat intake analysed as a specific nutrient or as a percentage of energy and risk of breast cancer. The present analysis and review updates the current state of the science and provides a comprehensive assessment of the literature. Additional analyses that focus on specific types of fatty acids, the timing of dietary exposures (for example, childhood, adolescence, premenopausal, postmenopausal) and subsequent disease (i.e. premenopausal diagnosis $v$. postmenopausal diagnosis), or analyses that evaluate associations based on hormone receptor status, may provide valuable insight to potential patterns of associations. Furthermore, improvements in dietary assessment methodology in cohort studies as well as examinations of dietary fat and breast cancer in randomised clinical trials may serve to clarify any possible relationships.

\section{Acknowledgements}

We thank Ms Colleen Cushing and Ms Bonnielin Sceurman for their contributions to the preparation of this paper.

The present review was partially funded by the Beef Checkoff, through the National Cattlemen's Beef Association (NCBA) and by the National Pork Board. NCBA and the National Pork Board did not contribute to the writing, analysis or interpretation of research findings.
All authors contributed to the conceptualisation, implementation and writing of the manuscript. All authors reviewed the manuscript and approved of submission.

The authors report no conflicts of interest.

\section{References}

1. Armstrong B \& Doll R (1975) Environmental factors and cancer incidence and mortality in different countries, with special reference to dietary practices. Int J Cancer 15, 617-631.

2. Lea AJ (1966) Dietary factors associated with death-rates from certain neoplasms in man. Lancet ii, 332-333.

3. Rose DP, Boyar AP \& Wynder EL (1986) International comparisons of mortality rates for cancer of the breast, ovary, prostate, and colon, and per capita food consumption. Cancer 58, 2363-2371.

4. Hursting SD, Thornquist M \& Henderson MM (1990) Types of dietary fat and the incidence of cancer at five sites. Prev Med 19, 242-253.

5. Carroll KK \& Khor HT (1975) Dietary fat in relation to tumorigenesis. Prog Biochem Pharmacol 10, 308-353.

6. Freedman LS, Clifford C \& Messina M (1990) Analysis of dietary fat, calories, body weight, and the development of mammary tumors in rats and mice: a review. Cancer Res 50, 5710-5719.

7. Smith-Warner SA \& Stampfer MJ (2007) Fat intake and breast cancer revisited. J Natl Cancer Inst 99, 418-419.

8. Howe GR, Hirohata T, Hislop TG, et al. (1990) Dietary factors and risk of breast cancer: combined analysis of 12 case-control studies. J Natl Cancer Inst 82, 561-569.

9. Boyd NF, Martin LJ, Noffel M, et al. (1993) A meta-analysis of studies of dietary fat and breast cancer risk. Br J Cancer 68, 627-636.

10. Boyd NF, Stone J, Vogt KN, et al. (2003) Dietary fat and breast cancer risk revisited: a meta-analysis of the published literature. Br J Cancer 89, 1672-1685.

11. Smith-Warner SA, Spiegelman D, Adami HO, et al. (2001) Types of dietary fat and breast cancer: a pooled analysis of cohort studies. Int J Cancer 92, 767-774.

12. Prentice RL, Caan B, Chlebowski RT, et al. (2006) Low-fat dietary pattern and risk of invasive breast cancer: the Women's Health Initiative Randomized Controlled Dietary Modification Trial. JAMA 295, 629-642.

13. Cho E, Spiegelman D, Hunter DJ, et al. (2003) Premenopausal fat intake and risk of breast cancer. J Natl Cancer Inst 95, 1079-1085.

14. World Cancer Research Fund \& American Institute for Cancer Research (2007) Food, Nutrition, Physical Activity, and the Prevention of Cancer: A Global Perspective. Washington, DC: AICR.

15. Hunter DJ, Spiegelman D, Adami HO, et al. (1996) Cohort studies of fat intake and the risk of breast cancer - a pooled analysis. N Engl J Med 334, 356-361.

16. Kim EH, Willett WC, Colditz GA, et al. (2006) Dietary fat and risk of postmenopausal breast cancer in a 20-year follow-up. Am J Epidemiol 164, 990-997.

17. Wakai K, Tamakoshi K, Date C, et al. (2005) Dietary intakes of fat and fatty acids and risk of breast cancer: a prospective study in Japan. Cancer Sci 96, 590-599.

18. Sieri S, Krogh V, Muti P, et al. (2002) Fat and protein intake and subsequent breast cancer risk in postmenopausal women. Nutr Cancer 42, 10-17.

19. Thiébaut AC, Kipnis V, Chang SC, et al. (2007) Dietary fat and postmenopausal invasive breast cancer in the National 
Institutes of Health-AARP Diet and Health Study cohort. J Natl Cancer Inst 99, 451-462.

20. Michels KB, Rosner BA, Chumlea WC, et al. (2006) Preschool diet and adult risk of breast cancer. Int J Cancer 118, 749-754.

21. Frazier AL, Li L, Cho E, et al. (2004) Adolescent diet and risk of breast cancer. Cancer Causes Control 15, 73-82.

22. Howe GR, Friedenreich CM, Jain M, et al. (1991) A cohort study of fat intake and risk of breast cancer. $J$ Natl Cancer Inst 83, 336-340.

23. Voorrips LE, Brants HA, Kardinaal AF, et al. (2002) Intake of conjugated linoleic acid, fat, and other fatty acids in relation to postmenopausal breast cancer: the Netherlands Cohort Study on Diet and Cancer. Am J Clin Nutr 76, 873-882.

24. Mills PK, Beeson WL, Phillips RL, et al. (1989) Dietary habits and breast cancer incidence among Seventh-Day Adventists. Cancer 64, 582-590.

25. Graham S, Zielezny M, Marshall J, et al. (1992) Diet in the epidemiology of postmenopausal breast cancer in the New York State Cohort. Am J Epidemiol 136, 1327-1337.

26. Holmes MD, Hunter DJ, Colditz GA, et al. (1999) Association of dietary intake of fat and fatty acids with risk of breast cancer. JAMA 281, 914-920.

27. Greenland S \& Longnecker MP (1992) Methods for trend estimation from summarized dose-response data, with applications to meta-analysis. Am J Epidemiol 135, $1301-1309$.

28. Rothstein H, Sutton A \& Borenstein M (2005) Publication Bias in Meta-Analysis: Prevention, Assessments, and Adjustments. Chichester, UK: John Wiley and Sons, Ltd.

29. Hermann S, Linseisen J \& Chang-Claude J (2002) Nutrition and breast cancer risk by age 50: a population-based casecontrol study in Germany. Nutr Cancer 44, 23-34.

30. Richardson S, Gerber M \& Cenee S (1991) The role of fat, animal protein and some vitamin consumption in breast cancer: a case control study in southern France. Int J Cancer 48, 1-9.

31. Iscovich JM, Iscovich RB, Howe G, et al. (1989) A casecontrol study of diet and breast cancer in Argentina. Int $J$ Cancer 44, 770-776.

32. Hirohata T, Shigematsu T, Nomura AM, et al. (1985) Occurrence of breast cancer in relation to diet and reproductive history: a case-control study in Fukuoka, Japan. Natl Cancer Inst Monogr 69, 187-190.
33. Ferraroni M, Decarli A, Willett WC, et al. (1991) Alcohol and breast cancer risk: a case-control study from northern Italy. Int J Epidemiol 20, 859-864.

34. Potischman N, Coates RJ, Swanson CA, et al. (2002) Increased risk of early-stage breast cancer related to consumption of sweet foods among women less than age 45 in the United States. Cancer Causes Control 13, 937-946.

35. Ceber E, Sogukpinar N, Mermer G, et al. (2005) Nutrition, lifestyle, and breast cancer risk among Turkish women. Nutr Cancer 53, 152-159.

36. Graham S, Marshall J, Mettlin C, et al. (1982) Diet in the epidemiology of breast cancer. Am J Epidemiol 116, 68-75.

37. Willett W (2008) Nutritional epidemiology. In Modern Epidemiology, pp. 580-598 [KJ Rothman, S Greenland and TL Lash, editors]. Philadelphia: Lippincott Williams \& Wilkins.

38. United States Department of Agriculture Agricultural Research Service (2007) USDA Nutrient Database for Standard Reference, Release 20. Nutrient Data Laboratory homepage. http://www.nal.usda.gov/fnic/foodcomp

39. Terry PD, Rohan TE \& Wolk A (2003) Intakes of fish and marine fatty acids and the risks of cancers of the breast and prostate and of other hormone-related cancers: a review of the epidemiologic evidence. Am J Clin Nutr 77, 532-543.

40. Ip C, Dong Y, Ip MM, et al. (2002) Conjugated linoleic acid isomers and mammary cancer prevention. Nutr Cancer $\mathbf{4 3}$, $52-58$.

41. Larsson SC, Bergkvist L \& Wolk A (2009) Conjugated linoleic acid intake and breast cancer risk in a prospective cohort of Swedish Women. Am J Clin Nutr 90, 556-560.

42. Willett W (1998) Nutritional Epidemiology, 2nd ed. New York: Oxford University Press.

43. Bingham SA, Luben R, Welch A, et al. (2003) Are imprecise methods obscuring a relation between fat and breast cancer? Lancet 362, 212-214.

44. Freedman LS, Potischman N, Kipnis V, et al. (2006) A comparison of two dietary instruments for evaluating the fat-breast cancer relationship. Int $J$ Epidemiol 35, 1011-1021.

45. Freedman LS, Kipnis V, Schatzkin A, et al. (2008) Methods of epidemiology: evaluating the fat-breast cancer hypothesis - comparing dietary instruments and other developments. Cancer J 14, 69-74. 